\title{
REGULAR POLYGON SOLUTIONS OF THE $N$-BODY PROBLEM
}

\author{
L. M. PERKO AND E. L. WALTER
}

\begin{abstract}
In 1772, Lagrange showed that three masses at the vertices of an equilateral triangle, rotating about their common center of mass with an appropriate angular velocity, describe a periodic solution of the three-body problem. In this paper it is shown that for $N \geq 4, N$ masses at the vertices of a regular polygon, rotating about their common center of mass with an appropriate angular velocity, describe a periodic solution of the $N$-body problem if and only if the masses are equal.
\end{abstract}

1. Introduction and results. For $N \geq 2$, the equations of motion of the planar $N$-body problem can be written in the form

$$
\ddot{z}_{k}=-\sum_{j \neq k} m_{j} \frac{z_{k}-z_{j}}{\left|z_{k}-z_{j}\right|^{3}}
$$

where $z_{k}$ is the complex coordinate of the $k$ th mass $m_{k}$ in an inertial coordinate system; cf. [5, p. 95]. In equation (1) and throughout this paper, unless otherwise restricted, all indices and summations will range from 1 to $N$.

Let $\rho_{k}$ denote the $N$ complex $N$ th roots of unity; i.e.,

$$
\rho_{k}=e^{2 \pi i k / N} \text {. }
$$

This equation will also serve to define $\rho_{k}$ for any real number $k$. The center of mass of $N$ masses $m_{k}$ located at the vertices $\rho_{k}$ of a regular polygon inscribed in the unit circle is then given by

$$
z_{0}=\sum_{j} m_{j} \rho_{j} / M
$$

where $M=\sum_{j} m_{j}$. The functions describing their rotation about $z_{0}$ with angular velocity $\omega$ are then given by

$$
z_{k}(t)=\left(\rho_{k}-z_{0}\right) e^{i \omega t}
$$

There is no loss of generality in assuming that the regular polygon is inscribed in the unit circle since the $N$-body problem (1) is invariant under the transformation $t \rightarrow t / a^{3 / 2}, z \rightarrow z / a$ which reduces the functions $a\left(\rho_{k}-z_{0}\right)^{i \omega t}$ to the functions defined in (3).

In 1772, Lagrange [2] established his "equilateral-triangle solutions" of the 3body problem. His result is equivalent to the following theorem; cf. [5, p. 94].

TheOREM (LAgrange). For $N=3$ and $m_{k}>0$ the functions $z_{k}(t)$ given by (3) with $\omega^{2}=M / 3 \sqrt{3}$ are a solution of the 3 -body problem (1).

Received by the editors July 9, 1984 and, in revised form, September 14, 1984.

1980 Mathematics Subject Classification. Primary 70F10; Secondary 34C25.

(C) 1985 American Mathematical Society $0002-9939 / 85 \$ 1.00+\$ .25$ per page 
The following result is established in this paper:

THEOREM 1. If, for $N \geq 2$, the functions $z_{k}(t)$ given by (3) are a solution of the $N$-body problem (1), it follows that $\omega^{2}=M \gamma / N$ where

$$
\gamma=\frac{1}{4} \sum_{j \neq N} \csc (\pi j / N) .
$$

Note that for $N=3$, formula (4) gives $\omega^{2}=M / 3 \sqrt{3}$, which agrees with Lagrange's result. Also, it is interesting to note that for $N \geq 2$, the number $\gamma$ defined by (4) is a positive algebraic number.

The main result of this paper is

THEOREM 2. For $N \geq 4$ and $m_{k}>0$ the functions $z_{k}(t)$ given by (3) with $\omega^{2}=M \gamma / N$ and $\gamma$ given by (4) are a solution of the $N$-body problem (1) if and only if $m_{1}=m_{2}=\cdots=m_{N}$.

Part of this theorem is well known, i.e., that $N$ equal masses at the vertices of a regular polygon, rotating about the center of the polygon with an appropriate angular velocity $\omega$ (determined by Theorem 1) describe a periodic solution of the $N$ body problem (1); cf. [7, p. 279]. The computation necessary to establish this result was apparently first carried out by Hoppe $[1]$ in 1879 . The converse, for $N=4$, i.e., that four masses at the vertices of a square, rotating about their common center of mass with an appropriate angular velocity $\omega$ (determined by Theorem 1) describe a periodic solution of the four-body problem only if the masses are equal, follows from the work of MacMillan and Bartky [4]; cf. [7, p. 278]. The converse for $N=5$ follows from the work of Williams [6] which extends the work of MacMillan and Bartky. The converse can also be proved by elementary algebraic methods for the first few integers greater than five; however, the proofs become increasingly more difficult as $N$ increases.

In this paper the above theorem is established for all $N \geq 4$ by using some basic properties of circulant matrices. It is a new result for regular polygon solutions of the $N$-body problem which has been suspected but never proved.

2. The proof. The proof of Theorem 2 uses the concept of a circulant matrix. Let $N \geq 2$. An $N \times N$ matrix $C=\left[c_{k j}\right]$ is called circulant if

$$
c_{k j}=c_{k-1, j-1}
$$

where $c_{0 j}$ and $c_{k 0}$ are identified with $c_{N j}$ and $c_{k N}$ respectively. Define the $N \times N$ circulant matrices $A, B$ and $C_{\alpha}$ as follows:

$$
\begin{gathered}
a_{k j}= \begin{cases}\left(1-\rho_{j-k}\right) /\left|1-\rho_{j-k}\right|^{3} & \text { for } j \neq k, \\
0 & \text { for } j=k,\end{cases} \\
b_{k j}=\rho_{j-k}, \\
C_{\alpha}=A+\alpha B
\end{gathered}
$$

where $\rho_{k}$ is given by (2) and $\alpha \in \mathbf{C}$. That $A, B$ and $C_{\alpha}$ are circulant is obvious since the subscript $(j-1)-(k-1)=j-k$ and $\rho_{0}=\rho_{N}$. 
The proof hinges on showing that certain eigenvalues of the circulant matrix $C_{\alpha}$ with $\alpha=\gamma / N$ and $\gamma$ defined by (4) are nonzero. This is accomplished using the general formulas for the eigenvalues $\lambda_{k}$ and the eigenvectors $\vec{v}_{k}$ of a circulant matrix $C=\left[c_{k j}\right]$,

$$
\begin{gathered}
\lambda_{k}=\sum_{j} c_{1 j} \rho_{k-1}^{j-1}, \\
\vec{v}_{k}=\left(\rho_{k-1}, \rho_{k-1}^{2}, \ldots, \rho_{k-1}^{N}\right)^{T},
\end{gathered}
$$

given for example in [3, p. 66]. It is interesting to note that the appearance of the $\rho_{k}$ in formulas (6) and (7) is a result of only the circulant property of $C$ and is not dependent upon the $\rho_{k}$ occurring in equations (5) defining $C_{\alpha}$.

The proofs of Theorems 1 and 2 are carried out in a sequence of lemmas which are stated in more generality than is necessary in that the $m_{k}$ and $\omega$ are allowed to be complex in some instances.

LEMMA 1. For $\vec{m}=\left(m_{1}, \ldots, m_{N}\right)^{T} \in \mathbf{C}^{N}$ and $\omega \in \mathbf{C}$, the functions $z_{k}(t)$ given by (3) are solutions of the $N$-body problem (1) if and only if

$$
\left(A+\left(\omega^{2} / M\right) B\right) \vec{m}=\omega^{2} \overrightarrow{1}
$$

where $A$ and $B$ are defined by (5) and $\overrightarrow{1}=(1, \ldots, 1)^{T} \in \mathbf{C}^{N}$.

Proof. Direct substitution into the differential equation (1) shows that the $z_{k}(t)$ are a solution of (1) if and only if

$$
\left(\rho_{k}-z_{0}\right) \omega^{2} e^{i \omega t}=\sum_{j \neq k} m_{j} \frac{\rho_{k}-\rho_{j}}{\left|\rho_{k}-\rho_{j}\right|^{3}} e^{i \omega t}
$$

or if and only if

$$
\sum_{j \neq k} m_{j} \frac{\rho_{k}-\rho_{j}}{\left|\rho_{k}-\rho_{j}\right|^{3}}+\frac{\omega^{2}}{M} \sum_{j} m_{j} \rho_{j}=\omega^{2} \rho_{k} .
$$

Multiplying both sides by $\rho_{N-k}$ and noting that $\left|\rho_{k}-\rho_{j}\right|=\left|\rho_{k}\right| \cdot\left|1-\rho_{j-k}\right|=$ $\left|1-\rho_{j-k}\right|$, and then using equation (2) shows that this last set of equations is equivalent to equation (8).

LEMMA 2. For any $\alpha \in \mathbf{C}$, the eigenvalue $\lambda_{1}$ of $C_{\alpha}$ is independent of $\alpha$ and satisfies

$$
\lambda_{1}=\sum_{j \neq N} \frac{1-\rho_{j}}{\left|1-\rho_{j}\right|^{3}}
$$

furthermore, $\lambda_{1}=\gamma$ where $\gamma$ is the positive number defined by (4) and $\vec{v}_{1}=\overrightarrow{1}$.

COROLlaRY. If $m_{1}=m_{2}=\cdots=m_{N}>0$, then $z_{0}=0$ and the functions $z_{k}(t)=\rho_{k} e^{i \omega t}$ with $\omega^{2}=m_{1} \gamma$ are a solution of the $N$-body problem (1).

The corollary follows since, by Lemma $2, \lambda_{1}=\gamma$, which is independent of $\alpha$, and $\vec{v}_{1}=\overrightarrow{1}$. In particular, for $\alpha=\gamma / N$,

$$
(A+(\gamma / N) B) \overrightarrow{1}=\gamma \overrightarrow{1} .
$$


Multiplying both sides by $m_{1}>0$ shows that $\vec{m}=\left(m_{1}, m_{1}, \ldots, m_{1}\right)^{T} \in \mathbf{R}^{N}$ is a solution to equation (8) with $\omega^{2}=m_{1} \gamma$. If $m_{1}=m_{2}=\cdots=m_{N}$ then $z_{0}=0$, since $\sum_{j} \rho_{j}=0$. The corollary is now proved by Lemma 1 .

This corollary establishes the well-known part of Theorem 2 stated in the introduction.

ProOF. From equations (5) and (6) and elementary properties of the $N$ th roots of unity,

$$
\lambda_{1}=\sum_{j \neq 1} \frac{1-\rho_{j-1}}{\left|1-\rho_{j-1}\right|^{3}} \rho_{0}^{j-1}+\alpha \sum_{j} \rho_{j-1} \rho_{0}^{j-1}=\sum_{j \neq N} \frac{1-\rho_{j}}{\left|1-\rho_{j}\right|^{3}}
$$

which is independent of $\alpha$. Since the $(N-j)$ th term in this last sum is the complex conjugate of the $j$ th term, $\lambda_{1}$ is real, so

$$
\lambda_{1}=\sum_{j \neq N} \frac{\operatorname{Re}\left(1-\rho_{j}\right)}{\left|1-\rho_{j}\right|^{3}}=\sum_{j \neq N} \frac{1-\cos (2 \pi j / N)}{[2-2 \cos (2 \pi j / N)]^{3 / 2}}=\frac{1}{4} \sum_{j \neq N} \csc (\pi j / N) .
$$

Thus $\lambda_{1}=\gamma$ defined by equation (4). That $\vec{v}_{1}=\overrightarrow{1}$ follows immediately from (7).

LEMMA 3. If $\vec{m}=\left(m_{1}, m_{2}, \ldots, m_{N}\right)^{T} \in \mathbf{C}^{N}$ is a solution of equation (8) then $\omega^{2}=M \gamma / N$.

ProOF. Since by equation (6), $\lambda_{1}=\sum_{j} c_{1 j}$, it follows from Lemma 2 that the sum of any row, and hence the sum of any column, of the circulant matrix $A+\left(\omega^{2} / M\right) B$ is given by $\lambda_{1}=\gamma$. Adding the $N$ equations in (8) then leads to

$$
\lambda_{1}\left(m_{1}+m_{2}+\cdots+m_{N}\right)=\omega^{2} N .
$$

This proves the lemma.

Lemmas 1 and 3 then establish Theorem 1 stated in the introduction.

LEMMA 4. The eigenvalue $\lambda_{N}$ of $A+(\gamma / N) B$ defined by (5) with $\gamma$ defined by (4) and $\lambda_{N}$ given by (6) satisfies $\lambda_{N}=0$.

ProOF. It follows from equations (5) and (6) and elementary properties of the $N$ th roots of unity that

$$
\begin{aligned}
\lambda_{N} & =\sum_{j \neq 1} \frac{1-\rho_{j-1}}{\left|1-\rho_{j-1}\right|^{3}} \rho_{N-1}^{j-1}+\frac{\gamma}{N} \sum_{j} \rho_{j-1} \rho_{N-1}^{j-1} \\
& =\sum_{j \neq N} \frac{1-\rho_{j}}{\left|1-\rho_{j}\right|^{3}} \bar{\rho}_{j}+\frac{\gamma}{N} N
\end{aligned}
$$

since $\rho_{N-1}^{j-1}=\bar{\rho}_{1}^{j-1}=\bar{\rho}_{j-1}$ and $\rho_{j} \bar{\rho}_{j}=\left|\rho_{j}\right|^{2}=1$. Thus by Lemma 2 ,

$$
\lambda_{N}=\sum_{j \neq N} \frac{\bar{\rho}_{j}-1}{\left|1-\rho_{j}\right|^{3}}+\gamma=-\bar{\lambda}_{1}+\gamma=-\lambda_{1}+\gamma=0 .
$$

LeMma 5. For $\alpha \in \mathbf{R}, C_{\alpha}$, defined by (5), is Hermitian.

PROOF. Since $C_{\alpha}$ is circulant, for $C_{\alpha}=\left[c_{k j}\right]$ it suffices to show that $\bar{c}_{1 j}=$ $c_{1, N-j+2}$. From equations (5), for $\alpha \in \mathbf{R}$,

$$
\bar{c}_{1 j}=\frac{1-\bar{\rho}_{j-1}}{\left|1-\rho_{j-1}\right|^{3}}+\alpha \bar{\rho}_{j-1}=\frac{1-\rho_{N-j+1}}{\left|1-\rho_{N-j+1}\right|^{3}}+\alpha \rho_{N-j+1}=c_{1, N-j+2} .
$$


From this point on $\lambda_{k}$ will denote the $N$ eigenvalues of the specific circulant matrix $C_{\gamma / N}=A+(\gamma / N) B$ with $A$ and $B$ defined by (5) and $\gamma$ defined by (4). Then, by Lemma $4, \lambda_{N}=0$.

The following two lemmas and their corollary form the basis for the proof of Theorem 2.

LEMMA 6. If $\lambda_{k} \neq 0$ for $k \neq N$ then any solution $\vec{m} \in \mathbf{C}^{N}$ of equation (8) has the form

$$
\vec{m}=(M / N) \overrightarrow{1}+a \vec{v}_{N}
$$

where $a \in \mathbf{C}$ and $\vec{v}_{N}=\left(\bar{\rho}_{1}, \bar{\rho}_{1}^{2}, \ldots, \bar{\rho}_{1}^{N}\right)^{T} \in \mathbf{C}^{N} ;$ furthermore, if $a \vec{v}_{N} \in \mathbf{R}^{N}$ then either $N=2$ or $a=0$.

PROOF. If $\vec{m} \in \mathbf{C}^{N}$ is any solution of (8), by Lemma $3, \omega^{2} / M=\gamma / N$, and then equation (8) has the form $(A+(\gamma / N) B) \vec{m}=\omega^{2} \overrightarrow{1}$. Since $A+(\gamma / N) B$ is Hermitian by Lemma 5 , its eigenvectors $\left\{\vec{v}_{1}, \ldots, \vec{v}_{N}\right\}$, given by (7), are a basis for $\mathbf{C}^{N}$. Hence $\vec{m}=\sum_{k} c_{k} \vec{v}_{k}$. Thus if $\vec{m}$ is a solution of (8),

$$
(A+(\gamma / N) B) \vec{m}=\sum_{k} c_{k} \lambda_{k} \vec{v}_{k}=\omega^{2} \overrightarrow{1} \text {. }
$$

By Lemma 4, $\lambda_{N}=0$, and by Lemmas 2 and $3, \omega^{2}=M \gamma / N=M \lambda_{1} / N$ and $\vec{v}_{1}=\overrightarrow{1} ;$ thus,

$$
\left(c_{1}-M / N\right) \lambda_{1} \vec{v}_{1}+\sum_{1 \neq k \neq N} c_{k} \lambda_{k} \vec{v}_{k}=\overrightarrow{0} .
$$

But because the eigenvectors are linearly independent, if $\lambda_{k} \neq 0$ for $k \neq N, c_{1}-$ $M / N=c_{2}=\cdots=c_{N-1}=0$. Thus $\vec{m}=(M / N) \overrightarrow{1}+a \vec{v}_{N}$ where $a=c_{N} \in \mathbf{C}$. To conclude the proof of the lemma note that, since $\rho_{1}^{N}=1$, from equation (7) it follows that $a \vec{v}_{N}=\left(a \bar{\rho}_{1}, a \bar{\rho}_{1}^{2}, \ldots, a \bar{\rho}_{1}^{N-1}, a\right)$ which is in $\mathbf{R}^{N}$ only if either $a=0$ or $\rho_{1} \in \mathbf{R}$; that is only if $a=0$ or $N=2$.

Note that the real solutions of equation (8) for $N=2$ correspond to the circular solutions of the two body problem with arbitrary masses.

LEMMA 7. For odd $N$, if $\lambda_{(N+1) / 2}=0$ and $\lambda_{k} \neq 0$ otherwise for $k \neq N$, then any solution $\vec{m} \in \mathbf{C}^{N}$ of equation (8) has the form

$$
\vec{m}=(M / N) \overrightarrow{1}+a \vec{v}_{N}+b \vec{v}_{(N+1) / 2}
$$

where $a, b \in \mathbf{C}, \vec{v}_{N}=\left(\bar{\rho}_{1}, \bar{\rho}_{1}^{2}, \ldots, \bar{\rho}_{1}^{N}\right)^{T} \in \mathbf{C}^{N}$ and

$$
\vec{v}_{(N+1) / 2}=\left(\bar{\rho}_{(N+1) / 2}, \bar{\rho}_{(N+1) / 2}^{2}, \ldots, \bar{\rho}_{(N+1) / 2}^{N}\right)^{T} \in \mathbf{C}^{N} \text {; }
$$

furthermore, if $a \vec{v}_{N}+b \vec{v}_{(N+1) / 2} \in \mathbf{R}^{N}$ then either $N=3$ or $a=b=0$.

PROOF. The first part is proved in exactly the same way as the first part of Lemma 6. To prove the second part, assume $N>3$ and let $r_{k}=a \bar{\rho}_{1}^{k}+b \bar{\rho}_{(N+1) / 2}^{k}$. Then from equation (7), $a \vec{v}_{N}+b \vec{v}_{(N+1) / 2} \in \mathbf{R}^{N}$ only if $r_{k} \in \mathbf{R}$ for all $k$. Since $\rho_{k}^{N-1}=\bar{\rho}_{k}$ and $\rho_{k}^{N}=1$, it follows that

$$
\begin{aligned}
r_{1}+r_{N-1} & =a\left(\bar{\rho}_{1}+\rho_{1}\right)+b\left(\bar{\rho}_{(N+1) / 2}+\rho_{(N+1) / 2}\right), \\
r_{N} & =a+b .
\end{aligned}
$$


That $a, b \in \mathbf{R}$ follows by applying Cramer's rule to this system whose determinant is nonzero. Using equation (2),

$$
\begin{aligned}
& r_{1}=a(\cos 2 \pi / N-i \sin 2 \pi / N)+b[\cos (N+1) \pi / N-i \sin (N+1) \pi / N], \\
& r_{2}=a(\cos 4 \pi / N-i \sin 4 \pi / N)+b[\cos 2(N+1) \pi / N-i \sin 2(N+1) \pi / N] .
\end{aligned}
$$

Since $r_{1}, r_{2}, a, b \in \mathbf{R}$, it follows that

$$
\begin{array}{r}
a \sin 2 \pi / N+b \sin (N+1) \pi / N=0, \\
a \sin 4 \pi / N+b \sin 2(N+1) \pi / N=0 .
\end{array}
$$

This system has a nonzero solution $(a, b)$ only if its determinant is zero; that is, only if

$$
\begin{aligned}
0 & =\sin ^{2} 2 \pi / N+\sin \pi / N \sin 4 \pi / N \\
& =2 \sin 2 \pi / N \sin \pi / N\left(2 \cos ^{2} \pi / N+\cos \pi / N-1\right) .
\end{aligned}
$$

This last quantity is zero only if $\cos \pi / N=1 / 2$ or -1 , which is impossible for $N>3$. Thus for odd $N \geq 3, a \vec{v}_{N}+b \vec{v}_{(N+1) / 2} \in \mathbf{R}^{N}$ only if $a=b=0$ or $N=3$. This completes the proof of Lemma 7.

Note that for $N=3$ there are positive solutions of equation (8) which correspond to Lagrange's equilateral triangular solution of the three-body problem with arbitrary masses.

The following corollary is an immediate consequence of Lemmas 6 and 7.

COROLlaRY. Let $N \geq 4$. If $\lambda_{k} \neq 0$ for $k \neq N$, except that $\lambda_{(N+1) / 2}=0$ for odd $N$, then $\vec{m}$ is a real solution of equation (8) only if $\vec{m}=(M / N) \overrightarrow{1}$, i.e., only if $m_{1}=m_{2}=\cdots=m_{N}$.

In view of Lemma 1 and the corollary to Lemma 2, in order to prove Theorem 2 , it only remains to prove that the hypotheses of the above corollary are satisfied. The following technical lemma is necessary for this task.

LEMMA 8.

$$
\sum_{j} \rho_{1}^{j k}= \begin{cases}0 & \text { for } k \neq N \\ N & \text { for } k=N\end{cases}
$$

PROOF. For $k=N, \rho_{1}^{j N}=1$ and the result follows. For $k \neq N$, the following standard formulas are used:

$$
\begin{aligned}
& \sum_{j=0}^{n} \cos 2 j a=\frac{\sin [a(n+1)] \cos a n}{\sin a} \\
& \sum_{j=0}^{n} \sin 2 j a=\frac{\sin [a(n+1)] \sin a n}{\sin a}
\end{aligned}
$$

Then

$$
\sum_{j} \rho_{1}^{j k}=\sum_{j=0}^{N-1} \rho_{1}^{j k}=\sum_{j=0}^{N-1} \cos (2 \pi j k / N)+i \sum_{j=0}^{N-1} \sin (2 \pi j k / N) .
$$

The substitution of $N-1$ for $n$ and of $\pi k / N$ for $a$ in (9) and (10) shows that the last two sums are zero for $k \neq N$. 
LemMA 9. For any $\alpha \in \mathbf{C}$ and for $2 \leq k \leq N-1$, the eigenvalues $\lambda_{k}$ of $C_{\alpha}$ are independent of $\alpha$; furthermore, they are real and $\lambda_{k}=-\lambda_{N-k+1}$.

PROOF. From equations (5) and (6),

$$
\lambda_{k}=\sum_{j} c_{1 j} \rho_{k-1}^{j-1}=\sum_{j \neq 1} \frac{1-\rho_{j-1}}{\left|1-\rho_{j-1}\right|^{3}} \rho_{k-1}^{j-1}+\alpha \sum_{j} \rho_{j-1} \rho_{k-1}^{j-1} .
$$

By shifting the index $j$ in the first sum and by using $\rho_{1}^{0}=\rho_{1}^{N k}$ in the second sum,

$$
\lambda_{k}=\sum_{j \neq N} \frac{1-\rho_{j}}{\left|1-\rho_{j}\right|^{3}} \rho_{k-1}^{j}+\alpha \sum_{j} \rho_{1}^{j k} .
$$

Thus, by Lemma 8 ,

$$
\lambda_{k}=\sum_{j \neq N} \frac{1-\rho_{j}}{\left|1-\rho_{j}\right|^{3}} \rho_{k-1}^{j}
$$

which is independent of $\alpha$. But, by Lemma $5, \lambda_{k}$ is real if $\alpha$ is real. Thus $\lambda_{k}$ is real, independent of $\alpha$ being real. To complete the proof, note that $\left(1-\rho_{j}\right) \rho_{N-k}^{j}=$ $\left(1-\rho_{j}\right) \rho_{-k+1-1}^{j}=\left(\rho_{-1}^{j}-1\right) \rho_{-k+1}^{j}=-\left(1-\bar{\rho}_{j}\right) \bar{\rho}_{k-1}^{j}$. Then by $(11)$,

$$
\lambda_{N-k+1}=\sum_{j \neq N} \frac{1-\rho_{j}}{\left|1-\rho_{j}\right|^{3}} \rho_{N-k}^{j}=-\sum_{j \neq N} \frac{1-\bar{\rho}_{j}}{\left|1-\rho_{j}\right|^{3}} \bar{\rho}_{k-1}^{j}=-\bar{\lambda}_{k}=-\lambda_{k} .
$$

Corollary. For $\alpha \in \mathbf{C}, N \geq 3$, and $k \neq N$, the eigenvalues $\lambda_{k}$ of $C_{\alpha}$, defined by (5), are given by

$$
\lambda_{k}=\frac{1}{4} \sum_{j \neq N} \frac{\sin [\pi j(2 k-1) / N]}{\sin ^{2}(\pi j / N)} .
$$

PROOF. That (12) holds for $k=1$ follows from Lemma 2 and equation (4). For $2 \leq k \leq N-1$, it follows from (2) and elementary trigonometric identities that

$$
\frac{\operatorname{Re}\left[\left(1-\rho_{j}\right) \rho_{k-1}^{j}\right]}{\left|1-\rho_{j}\right|^{3}}=\frac{\sin [\pi j(2 k-1) / N]}{4 \sin ^{2}(\pi j / N)} .
$$

Substituting this result into equation (11) and using the fact that $\lambda_{k}$ is real leads to equation (12).

The next corollary follows by setting $k=(N+1) / 2$ in Lemma 9 .

Corollary. For odd $N, \lambda_{(N+1) / 2}=0$.

LEMMA 10. For even $N \geq 4, \lambda_{N / 2}>0$.

ProOF. By equation (12) and trigonometric identities,

$$
4 \lambda_{N / 2}=\sum_{j \neq N} \frac{\sin [\pi j(N-1) / N]}{\sin ^{2}(\pi j / N)}=\sum_{j \neq N}(-1)^{j-1} \csc (\pi j / N) .
$$

That this sum is positive is seen by noting that for $1 \leq j \leq N / 2$, the first summand is positive, the summands alternate in sign and their absolute values are decreasing. By symmetry about $j=N / 2$, the remaining part of the sum is also positive. 
LEMMA 11. For $N \geq 5$ and $2 \leq k \leq(N-1) / 2, \lambda_{k}-\lambda_{k-1}>\lambda_{k+1}-\lambda_{k}$.

PROOF. In equation (9), by substituting $q$ for $j, \pi j / N$ for $a$ and $k-1$ for $n$, and by using elementary trigonometry,

$$
\begin{aligned}
\sum_{q=0}^{k-1} \cos (2 \pi q j / N) & =\frac{\sin [\pi j k / N] \cos [\pi j(k-1) / N]}{\sin (\pi j / N)} \\
& =\frac{\sin [\pi j(2 k-1) / N]+\sin (\pi j / N)}{2 \sin (\pi j / N)} .
\end{aligned}
$$

Using this result with equation (12),

$$
\lambda_{k}=\frac{1}{4} \sum_{j \neq N}\left\{\frac{-1}{\sin (\pi j / N)}+\frac{2}{\sin (\pi j / N)} \sum_{q=0}^{k-1} \cos (2 \pi q j / N)\right\} .
$$

From this it follows that

$$
\begin{aligned}
\left(\lambda_{k}-\lambda_{k-1}\right)-\left(\lambda_{k+1}-\lambda_{k}\right) & =\frac{1}{2} \sum_{j \neq N} \frac{\cos [2 \pi j(k-1) / N]-\cos (2 \pi j k / N)}{\sin (\pi j / N)} \\
& =\sum_{j \neq N} \sin [\pi j(2 k-1) / N] .
\end{aligned}
$$

Now applying equation (10), by substituting $N-1$ for $n$ and $\pi(2 k-1) /(2 N)$ for $a$, and using more trigonometry,

$$
\begin{aligned}
\left(\lambda_{k}-\lambda_{k-1}\right)-\left(\lambda_{k+1}-\lambda_{k}\right) & =\frac{\sin [\pi(2 k-1) / 2] \sin [\pi(2 k-1)(N-1) /(2 N)]}{\sin [\pi(2 k-1) /(2 N)]} \\
& =\cot [\pi(2 k-1) /(2 N)] .
\end{aligned}
$$

This last expression is positive because $0<\pi(2 k-1) /(2 N)<\pi / 2$ for $2 \leq k \leq$ $(N-1) / 2$. $N$.

LEMMA 12. For $N \geq 4$ and $k \neq N, \lambda_{k} \neq 0$ except that $\lambda_{(N+1) / 2}=0$ for old

ProOF. To show $\lambda_{k}>0$ for $1 \leq k<N / 2$, suppose $\lambda_{k} \leq 0$. Then by Lemma 11, $\lambda_{k+1}+\lambda_{k-1}<2 \lambda_{k}$ which means either $\lambda_{k+1}<\lambda_{k}$ or $\lambda_{k-1}<\lambda_{k}$. If $\lambda_{k-1}<\lambda_{k}$ then, again by Lemma 11, $\lambda_{k-2}-\lambda_{k-1}<\lambda_{k-1}-\lambda_{k}<0$, which means $\lambda_{k-2}<$ $\lambda_{k-1}<\lambda_{k} \leq 0$. Proceeding by induction, $\lambda_{1}<0$ which contradicts Lemma 2. If $\lambda_{k+1}<\lambda_{k}$ a similar proof shows that, for odd $N, \lambda_{(N+1) / 2}<0$ which contradicts the second corollary to Lemma 9, or for even $N, \lambda_{N / 2}<0$ which contradicts Lemma 10. So $\lambda_{k}>0$ for $1 \leq k<N / 2$. That $\lambda_{k}<0$ for $N / 2<k \leq N-1$ follows from Lemma 9.

Finally, Lemma 12 shows that the hypotheses of the corollary to Lemmas 6 and 7 are satisfied and the proof of Theorem 2 is thus complete.

ACKNOWLEDGMENTS. The authors wish to express their sincere appreciation to Dr. P. J. Horn of the Department of Mathematics at Northern Arizona University for introducing them to circulant matrices and for suggesting their application to regular polygon solutions of the $N$-body problem. This work was partially supported by the National Science Foundation under Grant MCS 8201020 and by a Northern Arizona University Organized Research Grant. 


\section{REFERENCES}

1. R. Hoppe, Erweiterung der bekannten Speciallösung des Dreikörperproblems, Arch. Math. Phys. 64 (1879), 218.

2. J. Lagrange, Oeuvres, vol. 6, Gauthier-Villars, Paris, 1873; also in Prix de l'Académie, vol. IX, Ch. II, Panekoucke, Paris, 1777, p. 61.

3. M. Marcus and H. Minc, A survey of matrix theory and matrix inequalities, Allyn and Bacon, Boston, Mass., 1964.

4. W. D. MacMillan and W. Bartky, Permanent configurations in the problem of four bodies, Trans. Amer. Math. Soc. 34 (1932), 838-874.

5. C. L. Siegel and J. K. Moser, Lectures on celestial mechanics, Springer-Verlag, New York, Heidelberg, Berlin, 1971.

6. W. L. Williams, Permanent configurations in the problem of five bodies, Trans. Amer. Math. Soc. 44 (1938), 563-579.

7. A. Wintner, The analytical foundations of celestial mechanics, Princeton Univ. Press, Princeton, N.J., 1947.

Department of Mathematics, Northern Arizona University, Flagstaff, ARIZONA 86011 